\title{
Vestibular Assessment in Chronic Noise Exposure Subjects
}

Ali A. Ali ${ }^{1}$, Atef A. El-Maraghy ${ }^{2}$, Ahmed M. Ahmed ${ }^{1}$, Hany A. Mohany ${ }^{1}$

${ }^{1}$ Audiovestibular Unit, Department of Otolaryngology, Faculty of Medicine - Al-Azhar University

${ }^{2}$ Department of Otolaryngology, Faculty of Medicine - Al-Azhar University, Cairo, Egypt.

*Corresponding author: Hany A. Mohany, Mobile: (+20)1221971274, Email: hanyafm@yahoo.com

\begin{abstract}
Background: The damaging effect of noise on vestibular disturbance is well known, first described in 1890 by Haberman in tinkers with occupational hearing loss. Others have reported vestibular disturbances and abnormalities, such as balance disorders, dizziness, vertigo, and even spontaneous nystagmus in workers exposed to various kinds of occupational noise. Objective: To evaluate vestibular function in subjects with chronic noise exposure.

Subjects and methods: Eighty subjects were included in the study, divided into 2 groups: 60 subjects exposed to noise in laundry with mean age $41.53 \pm 11.15$ (study group) and 20 subjects not exposed to noise with mean age $38.60 \pm 6.48$ (Control group). All subjects underwent audiovestibular evaluations (puretone audiometry, tympanometry, vedionystagmography, and posturography).

Results: This study demonstrated elevated hearing threshold at audiometric frequencies $2-8 \mathrm{KHz}(\mathrm{pv}<\mathbf{0 . 0 0 1})$ and speech discrimination ( $\mathrm{pv}<\mathbf{0 . 0 0 1}$ ). in addition, marked caloric weakness and reduced SOT composite scores $(\mathrm{pv}=$ $<\mathbf{0 . 0 0 1}$ ), reduced SOT equilibrium scores in noise exposure subjects ( $\mathrm{pv}<\mathbf{0 . 0 0 1})$, reduced SOT sensory scores in VEST and PREF ( $\mathrm{Pv}<\mathbf{0 . 0 0 1})$. Furthermore, there was a positive correlation between duration of exposure and auditory and vestibular implications.
\end{abstract}

Conclusion: this study revealed apparent effect of noise on auditory and vestibular system.

Keywords: Noise, Auditory System, Vestibular System, Noise Induced Hearing Loss.

\section{INTRODUCTION}

Noise is defined as "unwanted sound" with various deleterious effects on health. The most significant physiological effect of exposure to noise is either temporary or permanent hearing loss. Partial disappearance of the organ of Corti was found with destruction of the hair cells, the extensive damage being in the lower basal coil ${ }^{(\mathbf{1})}$.

Unfortunately, noise-induced hearing loss (NIHL) is so common that a majority of the workers believe that it is part of their normal working life course. It has been estimated that 1.1 million people are exposed to excessive noise at work; among these, 0.17 million are predicted to suffer significant ear damage as a direct result of noise ${ }^{(2)}$.

The vestibular portion of the auditory system helps in maintaining balance in association with the ocular and the central nervous system. The vestibular end organs and the cochlea have a common evolutionary origin and utilize the same basic principle of mechano-electric transduction with the help of the sensory hair cells ${ }^{(3)}$.

The damaging effect of noise on vestibular disturbance is well known, first described in 1890 by Haberman in tinkers with occupational hearing loss. Others have reported vestibular disturbances and abnormalities, such as balance disorders, dizziness, vertigo, and even spontaneous nystagmus in workers exposed to various kinds of occupational noise ${ }^{(4)}$.

Aim of the work: To evaluate vestibular function in subjects with chronic noise exposure.

\section{SUBJECTS AND METHOD \\ Subjects:}

\section{Study group:}

This study was conducted at the Audiology Clinic, Al Hussein hospital, Faculty of Medicine, Al-Azhar university during the period from December 2014 to November 2018. It consisted of of 60 subjects exposed to noise working in laundry and its subgroups.

\section{Control group:}

Consistent of 20 normal adult volunteers, age and gender matched with patient group without history of otologic disorders or noise exposure.

\section{Exclusion criteria:}

No current or past history of general medical, neurologic or otologic findings known to adversely affect hearing or balance, compensate for balance related problems or interfere with test requirements.

\section{Equipment:}

1- Audiometer: interacoustic AC40.

2- Immittancemeter: Grason-Stadler GSI 39, Autotymp. Middle Ear analyzer.

3- Videonystagmography (VNG): Computarized 2channel VNG biomedical using monocular goggles. Micro-medical technologies Inc., spectrum software, chatham, Illinois, USA.

4- Computerized Dynamic Posturography (CDP): Neurocom Smart Equites, International. Inc., Clackamas, Oregon, USA.

5- Sound level meter (SLM): precision, Bruel \& KJaer type 2235.

6- Sound treated room, (locally made).

\section{Ethical consideration and Written informed consent :}

An approval of the study was obtained from AlAzhar University academic and ethical committee. Every patient signed an informed written consent for acceptance of the operation. 


\section{Methods:}

Both study and control groups were subjected to:

A detailed otological and neurological history with full description of dizziness, tinnitus, hearing loss and otalgia when presented.

$>$ Physical, otolaryngological, and neurological examination.

$>$ Audiological tests including:

- Pure tone audiometry (PTA): air conduction threshold was tested at frequencies $0.25,0.5,1,2,3,4,6$ and 8 $\mathrm{KHz}$. Bone conduction threshold was tested at frequencies between 500-4000, also at octave interval.

- Speech audiometry: Speech reception threshold (SRT), using Arabic spondiac ward ${ }^{(5)}$, and word discrimination score (WDS), using Arabic phonetically balanced (PB) words ${ }^{(6)}$.

- Acoustic immittance testing: This included tympanometry and acoustic reflexes (ipsilateral and contralateral) using probe tone frequency at $226 \mathrm{~Hz}$ and tone stimuli at 500, 1000, 2000, and $4000 \mathrm{~Hz}$.

$>$ Vestibular tests including:

- Videonystagmography (VNG):

Calibration was done for accurate recording of eye movements.

- Spontaneous nystagmus, Gaze nystagmus, Saccade, Smooth pursuit, Optokinetic and caloric testes were done.

- The bithermal caloric testing was in the following sequence: right $\operatorname{cool}\left(30^{\circ} \mathrm{C}\right)$, left $\operatorname{cool}\left(30^{\circ} \mathrm{C}\right)$, right warm $\left(44^{\circ} \mathrm{C}\right)$ and left warm $\left(44^{\circ} \mathrm{C}\right)$, with head anteroflexed $30^{\circ}$ from the supine position. The irrigation last for 30 seconds using an open loop caloric irrigator with $250 \mathrm{cc}$ water.

Data were interpreted in terms of Directional Preponderance (DP) and Unilateral Weakness (UW), which were considered significant when greater than $25 \%$ and $20 \%$, respectively ${ }^{(7)}$.

- Sensory organization test (SOT) of Computerized Dynamic Posturography (CDP):

The subject standing on a force plate, enclosed by a visual surround, estimating the position of the body sway. Subjects are exposed to six sensory conditions,

- SOT 1: Eyes open, steady surface and visual surround.

- SOT 2: Eyes closed, steady surface and visual surround.

- SOT 3: visual surround moved, eyes open and platform steady.

- SOT 4: Visual surround steady, platform moved and eyes open.

- SOT 5: Visual surround steady, platform moved and eyes closed.
- SOT 6: Platform and visual surround moved with eyes open. Condition 5 and 6 scores best represent the vestibular part of the overall balance system. A fall under any of the conditions $=$ a score of $0 \%$ and a score of $100 \%$ when no sway at all ${ }^{(\mathbf{8})}$.

Equilibrium scores (ES): a total of 18 scores were obtained, 3 for each of the 6 conditions. The ES of each condition is the arithmetic mean of its 3 trials.

Composite score (CS): is the mean overall score of the 18 test scores.

Sensory analyses (SA): identifies the sensory dysfunction that contributed to the overall SOT abnormality when the composite score falls within the abnormal range:

Somatosensory ratio (SOM) (condition $2 /$ condition 1) : measures ability to use somatosensory information.

Visual ratio (VIS) (condition 4/condition 1: measures ability to use visual information.

Vestibular ratio (VEST) (condition 5/ condition 1): measures ability to use vestibular information.

Preference ratio (PREF) (condition 3+6/ condition

2+5): measures reliance of visual information.

\section{Statistical analysis:}

Recorded data were analyzed using the statistical package for social sciences, version 20.0 (SPSS Inc., Chicago, Illinois, USA). Quantitative data were expressed as mean \pm standard deviation (SD). Qualitative data were expressed as frequency and percentage.

\section{The following tests were done:}

- Independent-samples t-test of significance was used when comparing between two means.

- Chi-square $\left(x^{2}\right)$ test of significance was used in order to compare proportions between two qualitative parameters.

- The confidence interval was set to $95 \%$ and the margin of error accepted was set to $5 \%$. The p-value was considered significant as the following:

- Probability (P-value)

- P-value $<0.05$ was considered significant.

- P-value <0.001 was considered as highly significant.

- P-value $>0.05$ was considered insignificant.

\section{RESULTS}

This study demonstrated elevated hearing threshold at audiometric frequencies $2-8 \mathrm{KHz}(\mathrm{pv}$ $<\mathbf{0 . 0 0 1}$ ) and speech discrimination ( $\mathrm{pv}<\mathbf{0 . 0 0 1}$ ).

There is statistically significant difference between cases and controls along pure tone thresholds except at 250,500 and $1000 \mathrm{~Hz}$ in both ears, with less thresholds and better discrimination in controls than in cases (Table 1). 
Table (1): comparison of pure tone thresholds and speech discrimination between cases and controls

\begin{tabular}{|l|c|c|c|c|}
\hline Variable & $\begin{array}{c}\text { Case group } \\
\text { (meangroup\&SD) }\end{array}$ & pntrol group (mean\&SD) & t- test & P. value \\
\hline Rt. 250 Hz & $12.83 \pm 4.05$ & $12.41 \pm 2.51$ & 0.67 & 0.500 \\
\hline Rt. 500 Hz & $14.3 \pm 3.32$ & $15.00 \pm 0.00$ & 0.78 & 0.450 \\
\hline Rt. 1000 Hz & $19.41 \pm 4.22$ & $20.00 \pm 0.00$ & 1.06 & 0.287 \\
\hline Rt. 2000 Hz & $17.25 \pm 5.32$ & $12.58 \pm 2.51$ & 6.13 & $0.001^{*}$ \\
\hline Rt. 3000 Hz & $35.1 \pm 22.76$ & $17.5 \pm 2.5$ & 6.1 & $0.001^{*}$ \\
\hline Rt. 4000 Hz & $37.83 \pm 25.46$ & $17.58 \pm 2.51$ & 6.12 & $0.001^{*}$ \\
\hline Rt. 6000 Hz & $44.75 \pm 24.70$ & $15 \pm 0$ & 8.3 & $0.001^{*}$ \\
\hline Rt. 8000 Hz & $47.16 \pm 26.51$ & $15.00 \pm 0.00$ & 9.39 & $0.001^{*}$ \\
\hline Lt. 250 Hz & $12.91 \pm 4.14$ & $12.41 \pm 2.51$ & 0.79 & 0.426 \\
\hline Lt. 500 Hz & $13.9 \pm 3.35$ & $14.16 \pm 1.87$ & 4.19 & 0.386 \\
\hline Lt. $1000 ~ H z$ & $18.75 \pm 4.28$ & $20.00 \pm 0.00$ & 2.26 & 0.026 \\
\hline Lt. 2000 Hz & $17.16 \pm 5.23$ & $12.58 \pm 2.51$ & 6.10 & $0.001^{*}$ \\
\hline Lt. 3000 Hz & $34.55 \pm 21.73$ & $17.5 \pm 2.5$ & 6.1 & $0.001^{*}$ \\
\hline Lt. 4000 Hz & $37.66 \pm 25.31$ & $17.58 \pm 2.51$ & 6.11 & $0.001^{*}$ \\
\hline Lt. 6000 Hz & $45.25 \pm 24.48$ & $14.25 \pm 1.78$ & 8.21 & $0.001^{*}$ \\
\hline Lt. 8000 Hz & $47.16 \pm 26.51$ & $15.00 \pm 0.00$ & 9.39 & $0.001^{*}$ \\
\hline Rt. WD\% & $92.66 \pm 5.05$ & $96.93 \pm 3.24$ & 5.50 & $0.001^{*}$ \\
\hline Lt. WD\% & $92.66 \pm 5.16$ & $96.93 \pm 3.24$ & 5.42 & $0.001^{*}$ \\
\hline
\end{tabular}

* Difference is statistically significant $(\mathrm{P}$ value $<0.05$ )

The study revealed that there is highly significant difference between cases and control across unilateral weakness and directional preponderance (Figure 1).

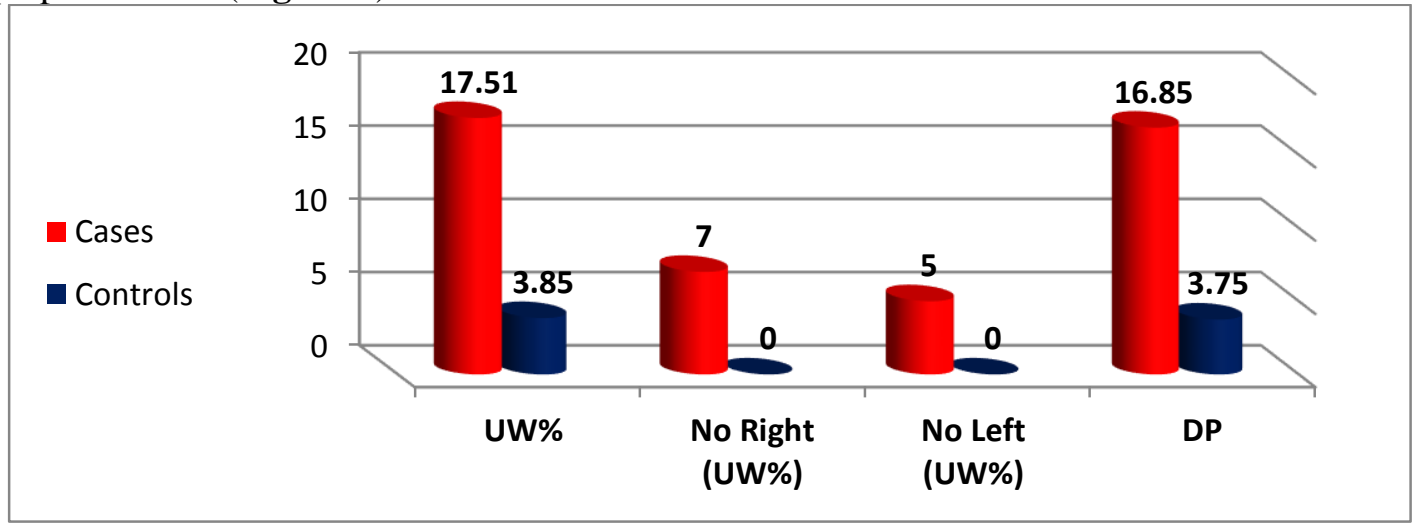

Figure (1): Unilateral weakness (UW\%) and directional preponderance (DP) in cases and controls There is significant difference in SOT composite scores between cases and controls (Figure 2).

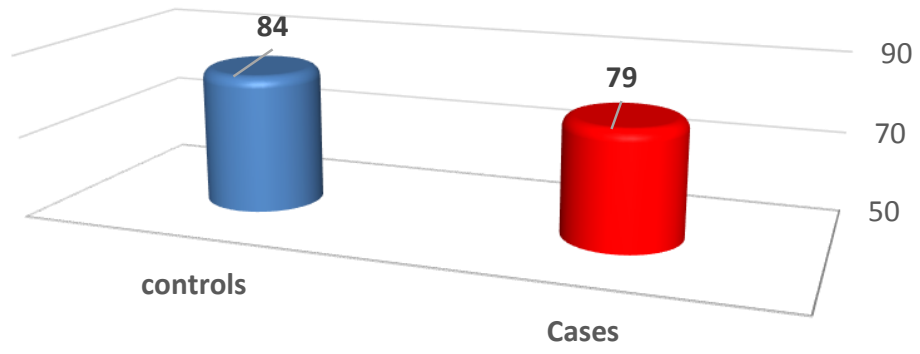

Figure (2): SOT composite score in the study and control groups.

The study revealed that there is significant difference in SOT sensory scores between cases and controls in VEST and PREF scores (Figure 3). 


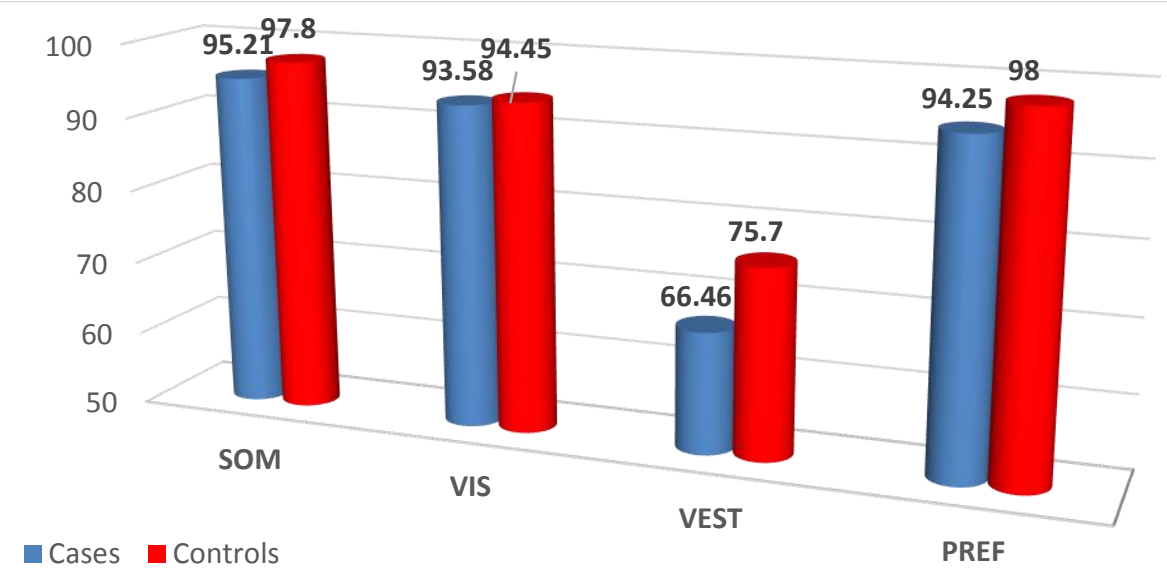

Figure (3): SOT sensory scores in the study and control groups

Table (2): SOT along the three groups of cases according to duration of exposure

\begin{tabular}{|l|l|l|l|l|l|}
\hline variable & Group 1 & Group 2 & Group 3 & ANOVA & P value \\
\hline Composite Score & $82.75 \pm 3.81$ & $78.33 \pm 3.68$ & $73.4 \pm 4.15$ & 28.28 & 2.94 \\
\hline C1. Eq. & $95.13 \pm 0.343$ & $93.4 \pm 1.3$ & $92.55 \pm 1.11$ & 29.62 & 1.51 \\
\hline C2. Eq. & $93.4 \pm 1.46$ & $90.33 \pm 1.69$ & $88.4 \pm 1.71$ & 49.35 & 3.64 \\
\hline C3. Eq. & $93.1 \pm 1.01$ & $89.3 \pm 1.25$ & $87.6 \pm 4.44$ & 21.52 & 1.08 \\
\hline C4. Eq. & $81.7 \pm 5.95$ & $79.16 \pm 3.2$ & $76.7 \pm 7.73$ & 3.39 & $0.040^{*}$ \\
\hline C5. Eq. & $69.5 \pm 6.84$ & $66.6 \pm 9.26$ & $59.2 \pm 11.5$ & 3.28 & $0.044^{*}$ \\
\hline C6. Eq. & $66.4 \pm 5.75$ & $62.7 \pm 6$ & $58.25 \pm 13.14$ & 3.24 & $0.050^{*}$ \\
\hline SOM & $96.4 \pm 1.07$ & $94.8 \pm 1.32$ & $91.4 \pm 2.1$ & 53.18 & 9.25 \\
\hline VIS & $95.9 \pm 1.47$ & $94.4 \pm 1.64$ & $90.6 \pm 1.46$ & 63.43 & 3.19 \\
\hline VEST & $72.87 \pm 9.62$ & $66.6 \pm 4.25$ & $57.35 \pm 13.6$ & 8.48 & $0.001^{* *}$ \\
\hline PREF & $96.2 \pm 1.27$ & $92.88 \pm 1.76$ & $90.9 \pm 1.57$ & 61.53 & 5.78 \\
\hline
\end{tabular}

$* *=$ high significant $<0.001$

There is highly significant change of posturography tests along with time exposure.

\section{DISCUSSION}

Exposure to noise can have adverse effects on hearing and balance mechanisms. Damage to the vestibular system, is a potential problem with a cochlear-damaging effect of loud sound ${ }^{(3)}$.

The study revealed that there is significant elevation of pure tone thresholds along audiometric frequencies 2, 3, 4, 6 and $8 \mathrm{KHz}$ in both sides and significant decrease in speech discrimination scores in both ears (Table 1). The results agreed with Sam $\boldsymbol{e t}$ al. (9) who reported an increase in pure tone audiometric thresholds from 250 to $8000 \mathrm{~Hz}$ in noise exposed subjects.

In the current study, during the vestibular evaluation of cases and controls, neither spontaneous nor positional nystagmus was detected via VNG recordings; there was caloric weakness that was statistically significant with mean UW\% of 17.5 for cases (12 cases with UW\% more than $25 \%, 7$ cases (58\%) with right UW \& 5 cases (42\%) with left UW) compared to 3.8 for that of control subjects. Also, directional preponderance was significantly higher for case than that for controls with mean DP\% of 16.85 for cases compared to 3.75 for that of control subjects that was statistically significant. Caloric weakness was found in 12 subjects of cases that represent $20 \%$ of cases (Figure 1).

The results agrees with Wang and Young ${ }^{(10)}$ who reported caloric weakness, canal paresis and VEMP abnormalities in chronic noise exposure patients but they reported a higher percentage of abnormalities that reaches $70 \%$ of cases

Zeigelboim et al. ${ }^{(11)}$ concluded that vestibular alterations found in the caloric test, and prevalence of alterations for the peripheral vestibular system with a predominance of irritative vestibular dysfunction. Dizziness was the most significant symptom in correlation of the vestibular test with neurotologic symptoms.

Shupak et al. ${ }^{(12)}$ reported a symmetrical centrally compensated decrease in the vestibular end organ response which is associated with the symmetrical hearing loss measured in the study group. Statistically significant correlations were found between the average hearing loss, the decrement in the average vestibulo-ocular reflex gain, and ENG caloric lateralization. These correlations might indicate a single mechanism for both cochlear and vestibular noise-induced injury. The results imply subclinical, 
well compensated malfunction of the vestibular system associated with NIHL.

As the exposure to noise increased, there was an increase in severity of dizziness. This is another supporting factor indicating that noise is the cause of dizziness in these workers. Another factor supporting the hypothesis is that in most instances, reduced hearing sensitivity and tinnitus are positively correlated. Coexistence of vestibular and otological symptoms strongly indicates that the vestibular and cochlear structures are gradually degenerating together due to chronic exposure to noise ${ }^{(13)}$.

In the current study, the mean SOT composite score for cases was significantly lower than that of the control subjects (Figure 2). There was significant reduction of SOT composite scores along all conditions except condition 1 . There was significant difference in SOT sensory scores between cases and controls specially vestibular score (Figure 3). Also, there was significant reduction of SOT along the three groups of cases according to duration of exposure (Table 2).

Industrial workers chronically exposed to various kinds of occupational noise, especially those with NIHL, often suffer from balance disorders such as dizziness, vertigo, and even spontaneous nystagmus. Saccular stimulation by loud sounds appears to be the underlying cause of the vestibular symptoms following loud sound exposure. Saccular macula is preferentially activated by sound, and neurons originating from the saccular striola are particularly affected ${ }^{\left({ }^{(14)}\right.}$.

\section{CONCLUSION}

In general, it can be inferred that the differences found with exposure to noise suggest that posturography may help in the investigation of changes in body balance caused by exposure in environments with high sound pressure levels, as in other cases.

- Noise has general hazards (neurologic, cardiovascular, psychiatric, etc.)

- Noise has auditory and vestibular implication

- Chronic noise exposure has cumulative effect on auditory and vestibular system.

- Regular exposure to noise increase probability of auditory and vestibular affection.

\section{RECOMMENDATIONS}

1. Regular audiologic and vestibular assessment for chronic noise exposure subjects.

2. Application of safety rules for chronic noise exposure subjects.
3. General knowledge establishment about noise hazards for chronic noise exposure subjects.

4. Further vestibular research for chronic noise exposure subjects.

\section{REFERENCES}

1. Peter WA (1997): Noise and the ear. In Scott-Brown's Otolaryngology, Vol.2, 6th edition, London. ButterworthHeinemann, 11: 1-34.

2. South $\mathbf{T}$ (2004): Managing noise and vibration at work. Elsevier, https://books.google.com.eg/books

3. Eisen M, Limb C (2007): An Essential Guide to Hearing and Balance Disorders. Lawrence, Erlbaum Assoc Inc.

4. Golz A, Westerman S, Westerman L (2001): The effects of noise on the vestibular system. Am J Otolaryngol., 22, 190-196.

5. Soliman SM, Fathalla A, Shehata W (1985): Development of the Arabic Staggered Spondiac Words (SSW) Test. Proceeding of the 8th Annual Ain Shams Congress, https://www.researchgate.net/publication/333047948_A SSESSMENT_OF_SUBCLINICAL_INNER_EAR_DY SFUNCTION_IN_PSORIATIC_ARTHRITIS_PATIEN TS

6. Soliman S (1976): Speech discrimination audiometry using Arabic phonetically balanced wards. Ain Shams Med J., 27(1): 27-30.

7. Teggi R, Colombo B, Bernasconi L et al. (2009): Migrainous Vertigo: Results of Caloric Testing and Stabilometric Findings. American Headache Society,17: 26-46.

8. Nevin M, Gihan M, Mona A et al. (2010): Assessment of the vestibulo-spinal reflex in migraine patients. Egypt J Neurol Psychiat Neurosurg., 47: 67-74.

9. Sam P, Kelli L, George A (1998): Noise-induced hearing loss in young adults: the role of personal listening devices and other sources of leisure noise. Laryngoscope, 108:1832-1839.

10. Wang Y, Young Y (2007): Vestibular-evoked myogenic potentials in chronic noise-induced hearing loss. Otolaryngol Head Neck Surg., 137: 607-611.

11. Zeigelboim B, Gueber G, Silva $T$ et al. (2014): Vestibular findings in military band musicians. Int Arch Otorhinolaryngol., 18:122-127.

12. Shupak A, Bar-El E, Podoshin L et al. (1994): Vestibular findings associated with chronic noise induced hearing impairment. Acta Otolaryngol., 114:579-585.

13. Ogido R, Costa EA, Machado Hda C (2009): Prevalence of auditory and vestibular symptoms among workers exposed to occupational noise. Rev Saude Publica, 43:377-80.

14. Goldberg JM (2000): Afferent diversity and the organization of central vestibular pathways. Exp Brain Res., 130:277-297. 